\title{
Cisplatin Conjugated Silver Nanoparticles Enhance Anticancer Activity in Breast Cancer Cells
}

\author{
Sayuri Gounden ${ }^{1}$, Moganavelli Singh ${ }^{1}$ \\ ${ }^{1}$ Nano-Gene and Drug Delivery Group, Discipline of Biochemistry, School of Life Sciences, University of KwaZulu-Natal, \\ University Road, Westville, Private Bag X54001, Durban, South Africa \\ 214502149@stu.ukzn.ac.za; singhm1@ukzn.ac.za
}

\section{Extended Abstract}

Owing to its aggressiveness and lack of effective treatment, cancer has dismal prognosis with the number of incidences increasing at an alarming rate. The ideal form of cancer therapy has been highly sought out, however most lack sensitivity, specificity and affordability, making existing treatments ineffective. Drastic side effects of conventional anticancer therapy due to the high concentration of therapeutic drugs used, enforces the need for a treatment strategy that would maximize the efficacy of the drug on cancer cells, whilst minimizing the efficacy of the drug on healthy rapidly growing cells. Recent studies have highlighted the use of nanoparticles for delivery of anticancer agents to cancer cells as angiogenesis increases tumor permeability to nano-drug delivery vehicles. Amongst the nanoparticles being investigated, nanorization of silver is essentially one of the significant features for good conductivity, chemical stability, relative lower toxicity and outstanding therapeutic potential. Silver nanoparticles (AgNPs) have been explored as delivery vehicles of therapeutic material to the nucleus thereby targeting diseased cells.

In this study, silver nanoparticles synthesized using the Turkevich method, displayed high loading capacities [>80\% encapsulation of Cisplatin (CIS)] using a chitosan (CS) biopolymer. Successful conjugation, size distribution and morphology of the nanoparticles was assessed by UV-Spectrophotometry, Nanoparticle Tracking Analysis (NTA) and Transmission Electron Microscopy (TEM). A 72-hour drug release study showed that Cisplatin was rapidly released from the nanocomplex at low $\mathrm{pH}$, which is favourable as cancerous cells thrive in acidic environments compared to healthy cells that survive at $\mathrm{pH}$ 7.4. Cytotoxicity profiles of the CS-AgNP-CIS nanocomplexes using the Sulforhodamine (SRB) and MTT assays revealed significant cell death in the breast cancer cell lines, MCF-7 and SKBR-3 compared to the other cell lines viz., HEPG2, CaCo-2 and HEK293. The CS-AgNP-CIS nanocomplexes were evidently more effective than the free drug, exhibiting more than $50 \%$ cell death at lower concentrations. This could be due to these nanocomplexes being readily taken up by the tumor cells, whereas at higher concentrations the nanocomplexes tend to aggregate, increasing in size, therefore posing a challenge to traverse the cellular membrane. The results of the study demonstrate a near ideal delivery system encapsulating a potent drug that exhibits peak performance and selectivity against breast cancer cell lines compared to normal healthy cells without the need for a targeting moiety. Hence, the need for further investigations both in vitro and in vivo are warranted in order to provide a better understanding of the nature of the cytotoxicity of these nanocomplexes and how any cytotoxic effect on normal cells may be eliminated. Overall, the results of this study provides basic and crucial information for the development of other such delivery systems of therapeutic molecules for the future treatment of cancer and more specifically breast cancer. 